\title{
High Prevalence of Wilson's Diseases with Low Prevalence of Kayser-Fleischer Rings among Patients with Cryptogenic Chronic Liver Diseases in Bangladesh
}

\author{
Nuzhat Choudhury ${ }^{1}$, Shamshad B Quraishi ${ }^{2}$, AKM Atiqullah ${ }^{3}$, Md Sakirul Islam Khan ${ }^{4}$, Mamun Al Mahtab ${ }^{5}$, Sheikh MF Akbar ${ }^{6}$
}

\begin{abstract}
Background: Chronic liver disease (CLD) is common in Bangladesh; however, a major bulk remains as cryptogenic CLD as they remain devoid of known pathological agents leading to have a check of Kayser-Fleischer (K-F) rings for possible Wilson's disease (WD) and many of these patients develop complications such as cirrhosis of liver and hepatocellular carcinoma. However, there remains considerable proportions of CLD patients with undefined etiology (cryptogenic CLD) and these patients cannot be provided effective therapy based on etiological factors. Here, the proportion of WD among cryptogenic CLD patients in Bangladesh has been evaluated to improve the management of CLD and reduce complications.

Materials and methods: A total of 941 patients with cryptogenic CLD [negative for hepatitis viruses, alcohol, nonalcoholic fatty liver disease (NAFLD), drug, and autoimmunity] were enrolled in the study. To assess if they have been suffering from WD, the levels of copper in 24-hour urine were evaluated. Definitive WD was diagnosed when 24-hour urinary copper output was $>100 \mu \mathrm{g}$ and strongly indicative WD patients excreted $>40 \mu \mathrm{g}$ of copper in 24 hours.

Results: Out of 941 patients with cryptogenic CLD, 212 patients were diagnosed as definitive WD and 239 patients as strongly indicative WD on the basis of 24-hours copper excretion. The age distribution ranging of the patients varied from 1 year to 90 years. There was a male predominance. Considerable numbers of WD patients had previous history of jaundice. Kayser-Fleischer rings were mostly uncommon and detected in five patients with WD only.

Discussion: Wilson's disease is not a rare entity in Bangladesh; rather, it seems to be fairly common among CLD patients. A country-wide epidemiological survey should be conducted for diagnosis of WD in Bangladesh to provide a proper management strategy for these huge numbers of WD patients. In fact, most of the WD patients are unaware of their diagnosis and the general physicians are equally unaware of diagnosis and management of WD.
\end{abstract}

Keywords: Chronic liver disease, Urinary copper, Wilson's disease.

Euroasian Journal of Hepato-Gastroenterology (2019): 10.5005/jp-journals-10018-1299

\section{INTRODUCTION}

Chronic liver disease (CLD) represents a major public health problem around the world, especially among the developing and resource-constrained countries. Chronic liver disease represents a diverse pathological entity that involves several organs in addition to the liver. ${ }^{1,2}$ The etiological agents of CLD are highly versatile. The major bulk of CLD is caused by two hepatotropic viruses: hepatitis B virus (HBV) and hepatitis C virus (HCV). However, abuse of alcohol and usage of various drugs represent important etiological agents of CLD in different parts of the world. Nonalcoholic fatty liver diseases, a lifestyle-related pathological status, also induce CLD and their prevalence is progressing with epidemiological parameters. Although metabolic disorders such as alpha antitrypsin deficiencies induce CLD, the prevalence may not be so high.

The diagnosis of CLD as well as exploration of etiological agent(s) is highly important because management of CLD must be based on etiology of the CLD. Also, an early diagnosis is warranted as management of early CLD would prevent and negatively regulate progression of CLD to complications like cirrhosis of liver and hepatocellular carcinoma. With the advent of new investigation facilities and considerable insights about molecular and cellular mechanisms about CLD, diagnosis of CLD has been progressing in recent decades. Various new markers of HBV and HCV have unfolded many undiagnosed CLD patients and millions of these patients
${ }^{1}$ Department of Ophthalmology, Bangabandhu Sheikh Mujib Medical University, Dhaka, Bangladesh

${ }^{2,3}$ Chemistry Division, Atomic Energy Centre, Bangladesh Atomic Energy Commission, Dhaka, Bangladesh

${ }^{4}$ Department of Anatomy and Embryology, Graduate School of Medicine, Ehime University, Toon, Ehime, Japan

${ }^{5}$ Department of Hepatology, Bangabandhu Sheikh Mujib Medical University, Dhaka, Bangladesh

${ }^{6}$ Department of Pathology, Ehime University Proteo-Science Center, Ehime University Graduate School of Medicine, Ehime, Japan

Address reprint requests to: Nuzhat Choudhury, Department of Ophthalmology, Bangabandhu Sheikh Mujib Medical University, Dhaka, Bangladesh, Phone: +880 1819248454, e-mail: dr.nuzhatchoudhury@ gmail.com

How to cite this article: Choudhury N, Quraishi SB, Atiqullah AKM, et al. High Prevalence of Wilson's Diseases with Low Prevalence of KayserFleischer Rings among Patients with Cryptogenic Chronic Liver Diseases in Bangladesh. Euroasian J Hepato-Gastroenterol 2019;9(2):67-70.

Source of support: Nil

Conflict of interest: None

would be rescued from development of serious CLD-related complications. Drug-induced CLD is diagnosed more efficiently at present, and the drug-lymphocyte stimulation test has helped 
High Prevalence of WDs with Low Prevalence of K-Y Rings among Patients with Cryptogenic CLDs in Bangladesh

to find the proper drug that may cause liver injury. Autoimmune CLD can also be explored in sufficient details, although developing countries should do more to get these technologies. Diagnosis of NAFLD and NAFLD-related CLD has been progressed during the last two decades and general consensus of patients and physicians has developed about their serious public health impact.

Even with all these approaches and progressive developments about diagnosis of CLD, still the etiologies of considerable numbers of CLD patients could not be clarified. These patients with CLD who cannot be diagnosed by any of the above known criteria are regarded as a group "cryptogenic CLD." ${ }^{3-5}$ The presence and essence of cryptogenic CLD is even more in developing and resource-constrained countries mainly due to limited scientific development and inherent limitation of activities of the healthcare delivery system.

Bangladesh is a country of 170 million people with millions of patients with CLD. The patients with CLD represent one of the major pathologies among hospital attendees and admissions in Bangladesh. Although a properly designed epidemiological study about etiologies of CLD is yet to be accomplished in Bangladesh, clinical observations by all groups of physicians state that there remains a major bulk of CLD patients who are not attributable to viruses (HBV and HCV), NAFLD, alcohol (a country where alcohol consumption is uncommon), drugs, and possibly autoimmunity.

With the postulation that a considerable proportion with cryptogenic CLD may belong to Wilson's diseases (WD), we designed the present study to assess the prevalence of WD among CLD patients of Bangladesh. In fact, patients with WD have been reported from Bangladesh and it seems that the prevalence may be considerable. ${ }^{6-10}$ However, these communications have been limited regarding exploration of clinical features of diagnosed WD patients attending medical facilities and especially tertiary medical institutions. However, there have been no study about the real prevalence of WD among CLD patients in Bangladesh. Thus, the patients, policy makers, and physicians are unaware of the impact of WD in Bangladesh. Also, WD has become a matter of scholastic interest of the specialists in tertiary medical facilities. As the real prevalence is unknown, the liver organizations of Bangladesh as well as ophthalmic societies and neurological associations have not provided any guidelines for the management of WD in Bangladesh. Naturally, the policy makers are unable to develop appropriate strategy for containment of WD.

To have proper insights about prevalence of WD, close collaborations among hepatologists, ophthalmologists, neurologists, and heavy metal specialists are essential as patients with WD may attend in any of these specialists after experiencing presenting symptoms that may be liver-related complains, anomalies in eye, neurological problems, or symptoms arising for copper accumulation.

Recently, a scientific collaboration has been developed among Department of Hepatology, Bangabandhu Sheikh Mujib Medical University, Dhaka; Department of Ophthalmology, Bangabandhu Sheikh Mujib Medical University, Dhaka, Bangladesh; and Atomic Energy Center, Bangladesh Atomic Energy Commission, Dhaka, Bangladesh. Accordingly, the underlying study has been undertaken to assess the prevalence of WD among cryptogenic CLD patients in Bangladesh.

\section{Materials and Methods}

The study enrolled a total of 941 patients who were diagnosed as cryptogenic CLD by different hepatologists and internalists of Bangladesh. The patients represented from the entire country as they were referred to Dhaka, the capital of Bangladesh, by physicians of peripheral hospitals. They have been suffering from CLD as biochemical, imaging, and other diagnostic procedures affirmed the diagnosis of CLD. However, they were negative for markers of HBV and HCV so that they cannot be regarded as HBV- or HCV-related CLD. They have not been suffering from NAFLD as the diagnostic criteria of NAFLD did not brought such a diagnosis. The patients did not give any history of alcohol consumption and in fact due to the religious factor, alcoholic CLD is almost nonexisting in Bangladesh. History of drug abuse was discarded as much as possible, and there has been no history that can be attributed to drug-induced CLD. The patients also did not exhibit an autoimmune pathology.

Taken together the diagnostic features of these patients were supposed to have a cryptogenic CLD. Patients were referred to the Department of Ophthalmology, BSMMU, Dhaka, Bangladesh to have a check about the presence of Kayser-Fleischer rings. The patients were finally referred to Atomic Energy Center, Dhaka, Bangladesh, to have insights about levels of copper in the urine. The center estimated the levels of copper in patient's urine and the levels were optimized as excretion of copper in urine of 24 hours.

\section{Measurement of Urinary Copper}

The measurement of urinary cupper was done at the Atomic Energy Center of Dhaka by a method that has been described and optimized. ${ }^{11}$ Copper-free containers were supplied to the patients from the laboratory in order to collect the samples. The collected urine samples were acidified with concentrated sulfuric acid (E. Merck, Germany) for mineralization at least 30 minutes prior to analysis. Then, concentration of $\mathrm{Cu}$ in urine was determined using a Varian AA240 FS Atomic Absorption Spectrometer. Acetylene gas with $99.99 \%$ purity was used for flame. A hollow cathode lamp having a wavelength of $324.8 \mathrm{~nm}$ and a slit of $0.7 \mathrm{~nm}$ was used and operated according to the conditions recommended by the manufacturer. The working standard solutions were prepared daily by the appropriate dilution of the stock Cu standard solution. Moreover, analysis of blank, duplicate, and spike recovery of the analyte was also performed daily as a part of the internal quality control practices. The levels of copper in 24-hour urine were optimized and expressed as micrograms of copper/24 hours of urine.

\section{Evaluation of the Levels of 24-hour Urinary Copper Excretion and Wilson's Disease}

This study presented here is an epidemiological study and intended to screen patients with WD. A total of 941 patients attended to have diagnosis of their cryptogenic CLD. The only test that was feasible is the assessment of copper in urine and optimized that to copper excretion in 24-hours urine. As described by Ala et al., ${ }^{12}$ the level of 24-hour copper excretion of $100 \mu \mathrm{g}$ was regarded as "Definitive WD" and when the copper level was above $40 \mu \mathrm{g}$ in 24-hour urine, it was regarded as "Strongly Indicative of WD."

\section{Results}

A cohort of 941 patients was enrolled in the study. As described by Aly et al., total copper of $>100 \mu \mathrm{g} / \mathrm{dL}$ in 24 -hour urine was regarded as "Definitive Diagnosis of WD." We found that 212 patients had urinary copper excretion of $>100 \mu \mathrm{g}$ in 24 hours. These patients were enrolled as patients with WD and we accomplished further analysis. In 239 patients, the levels of copper were $>40 \mu \mathrm{g}$ but $<100 \mu \mathrm{g}$ in 24-hour urine and the patients were regarded as "Strongly 
High Prevalence of WDs with Low Prevalence of K-Y Rings among Patients with Cryptogenic CLDs in Bangladesh

Indicative for WD." For the rest 490 patients, the levels of copper in 24-hour urine were less than $40 \mu \mathrm{g}$ and they were discarded from further analysis of WD.

The age distribution of patients with cryptogenic CLD has been shown in Table 1 . About $47 \%$ patients with cryptogenic CLD were below the age of 20 years. This indicate that cryptogenic CLD is a serious public health problem as the patients would require management for several decades if they express CLD in their teen ages.

The age of the patients with definitive WD was $27.66 \pm 16.51$ years $(n=212)$. On the other hand, the age of strongly indicative WD patients was $24.02 \pm 15.54$ years $(n=239)$. In fact, there was specific implication of age in the diagnosis of WD as the patients had a wide range of age diversity (1-90 years). Usually, classical or viral CLD is rare and almost nil among the age group of below 5 years and seldom patients experience viral CLD below the age of 20 years. A male predominance was detected in both types of WD of Bangladeshi patients. Sixty patients with definitive WD and 59 patients with strongly indicative WD had previous history of jaundice.

The levels of copper in 24-hour urine showed considerable diversities among patients with WD (Table 2). The levels of excreted copper were more than $500 \mu \mathrm{g}$ in 142 patients with WD. On the other hand, most of the patients $(>70 \%)$ with strongly indicative WD had copper excretion of $>50 \mu \mathrm{g}$.

The symptoms of the patients varied considerably and a major bulk of patients had several serious symptoms. General weakness, fatigue, fever, stomach ache, vomiting, anorexia, abdominal distension, abdominal disorder, cough, and headache were complained by majority of the patients. In addition, some patients had serious complains, such as trembling body, unable to speak, distorted tongue movement, slurred speech, blood in coughs, dysuria, convulsion, and psychological anomalies, which also became presenting symptoms of several patients. Many of the patients had several serious complains. Even someone gave history of senselessness. Although the present study was not designed to compile these complains, as our main attempt was to assess the prevalence of WD among cryptogenic CLD, the patients presented with moderate to severe illnesses.

Table 1: Age distribution of presumptive cryptogenic chronic liver diseases

\begin{tabular}{lc}
\hline Age (years) & $\begin{array}{l}\text { Number of patients with } \\
\text { cryptogenic chronic liver diseases }\end{array}$ \\
\hline $1-5$ & 34 \\
$6-10$ & 144 \\
$11-20$ & 265 \\
$21-30$ & 207 \\
$31-40$ & 117 \\
$41-50$ & 94 \\
$>50$ & 80 \\
\hline
\end{tabular}

Table 2: Divergent levels of copper excretion in patients with definitive Wilson's disease

\begin{tabular}{ll}
\hline Copper levels & Number of patients with definitive WD \\
\hline$>1,000 \mu \mathrm{g} / 24$ hours & 49 \\
$500-1,000 \mu \mathrm{g} / 24$ hours & 30 \\
$200-500 \mu \mathrm{g} / 24$ hours & 63 \\
$101-200 \mu \mathrm{g} / 24$ hours & 70 \\
\hline
\end{tabular}

The prevalence of $\mathrm{K}-\mathrm{F}$ rings was not notable in these patients with WD. These were found in only five patients in this cohort. The exact cause underlying this is not known; however, this may be a characteristic feature of WD of Bangladesh.

\section{Discussion}

Wilson's disease is an autosomal recessive disorder of hepatocyte copper trafficking caused by impaired function of P-type adenosine triphosphatase (ATPase), encoded by the ATP7B gene located on chromosome $13 q 14$ and consists of 21 exons. ${ }^{13,14}$ The disease usually affects the liver, but the clinical presentations may be diverse. Due to lack of study from developing countries, the typical clinical features could not be substantiated yet. Study from India has shown that the presentations of WD were mainly neurogenic and psychotic, whereas hepatic manifestations were seen in about 15\% of WD patients. They stated that out of 282 patients of WD, the following presentation was found: 42 (14.9\%); hepato-neurologic, $10(3.5 \%)$; neurologic, 195 (69.1\%); pure psychiatric, 7 (2.4\%); osseomuscular, $6(2.1 \%)$; and "presymptomatic," $15(5.3 \%))^{13}$ On the other hand, analyses of clinical symptoms in 100 Bangladeshi children showed that WD patients presented mainly with liver-related symptoms (CLD in $42 \%$, CLD with portal hypertension in $34 \%$, and acute hepatitis in $20 \%) .{ }^{9}$ But, both of these studies mainly described about WD diseases in children, and the major bulk or all patients in their cohort have been child. From these observations, it is apparent that a huge proportion of children has been suffering from an intractable disease like WD; however, due to lack of epidemiological studies, almost nothing is clear about its prevalence and management strategy.

The study presented here has not only unfolded the relative proportion of WD patients in Bangladesh, but it has also provided insights about several other important variables in public health sectors. The first, this is the first epidemiological study regarding WD in Bangladesh that has been possible due to an intense collaboration among ophthalmologists, hepatologists, and nuclear scientists. This will provide an impetus for future studies of these nature as Bangladesh lacks country-wide epidemiological data of almost all pathological conditions. The next, this study has unfolded a serious public health problem about cryptogenic CLD, a diagnostic area that also has not been properly addressed in Bangladesh. Out of 941 patients with cryptogenic CLD, 443 patients had an age of below 20 years and 178 patients were below 10 years of age. On one hand, prevalence of CLD in younger ages predicts a possible diagnosis of WD. On the other hand, about 47\% patients with cryptogenic CLD have age of below 20 years. They have already developed CLD and would possibly develop cirrhosis or liver and hepatocellular carcinoma in one or two decades. These patients should be treated on an emergency basis so that further progression of liver diseases can be contained. ${ }^{15}$

This is an epidemiological analysis and the study was not designed to evaluate different clinical features of Bangladeshi WD. We could not accomplish other investigations to support the diagnosis. Also, considerable numbers of these patients had previous bouts of jaundice as has been reported in India. ${ }^{16}$

If the global prevalence of WD is analyzed, it is found to be highly variable. The $\mathrm{WHO}$ estimates that the global prevalence of WD is $1 / 10,000$ to $1 / 30,000$ among general population. ${ }^{16}$ The prevalence of WD is higher in China than in the West. And, that may be relevant in other Asian countries. Very high prevalence of WD has been reported from Crete (1 WD among 17 people). If we consider the prevalence of WD would be around 1 in 10,000 in Bangladesh, 
there will be about 17,000 patients of WD in Bangladesh. A major bulk of these population would be children and proper diagnosis and treatment should be ensured for these patients.

As a routine matter, $\mathrm{HBV}, \mathrm{HCV}$, and NAFLD are checked when a patient with possible CLD attend physicians. Some tertiary hospitals and the advanced medical centers also check about autoimmune hepatitis, history of drug consumption, and some other etiologies of CLD. However, inquiry about WD and investigations to substantiate or negate WD are not accomplished in Bangladesh. Strategies should be adopted immediately to diagnose WD in Bangladesh.

The present study has some limitations as those are found in any study of this nature arising from developing and resource-constrained countries. We have not checked the genetic factor in these patients. Also, the patients could not be followed up about their clinical conditions. The diagnosis was mainly based on absence of other etiologies of CLD and presence of increased excretion of copper in urine. This simple process provides the easiest manner of an epidemiological study to get an idea about seriousness of that pathological condition. The absence of $\mathrm{K}-\mathrm{F}$ rings in most patients indicate the importance of copper estimation for diagnosis of WD in Bangladesh. Also, it remains to analyze if this is a characteristic feature of WD in this region. If this pattern prevails in other studies from Bangladesh or neighboring countries, more importance should be given to this important issue. As the presence of $\mathrm{K}-\mathrm{F}$ rings could not be explained by the age factor, more studies would be required to resolve this issue.

It is of interest to note that in an Indian cohort of WD, K-F rings have been found in about $90 \%$ patients with WD. However, the prevalence of $\mathrm{K}-\mathrm{F}$ rings was considerably low among patients with hepatic type of WD. ${ }^{17}$ In Indian series, 5 of 287 patients with WD had K-F rings, which implies with our data. In our series, most of the patients had hepatic manifestations and this may be a factor related to low prevalence of $\mathrm{K}-\mathrm{F}$ rings in this series. It is not sure if these patients would develop K-F rings in future or not. However, these should be followed in these patients.

Taken together, the study presented here has shown that estimation of copper in urine may be an early assessment procedure of thousands of patients with possible WD in Bangladesh and other developing and resource-constrained countries.

\section{References}

1. Asrani SK, Devarbhavi H, Eaton J, et al. Burden of liver diseases in the world. J Hepatol 2019;70(1):151-171. DOI: 10.1016/j.jhep.2018.09.014.
2. Yang JD, Hainaut $P$, Gores GJ, et al. A global view of hepatocellular carcinoma: trends, risk, prevention and management. Nat Rev Gastroenterol Hepatol 2019;16(10):589-604. DOI: 10.1038/s41575019-0186-y.

3. Nalbantoglu I, Jain D. Cryptogenic cirrhosis: old and new perspectives in the era of molecular and genomic medicine. Semin Diagn Pathol 2019;36(6):389-394. DOI: 10.1053/j.semdp.2019.07.003.

4. Mercado-Irizarry A, Torres EA. Cryptogenic cirrhosis: current knowledge and future directions. Clin Liver Dis (Hoboken) 2016;7(4):69-72. DOI: 10.1002/cld.539.

5. Czaja AJ. Cryptogenic chronic hepatitis and its changing guise in adults. Dig Dis Sci 2011;56(12):3421-3438. DOI: 10.1007/s10620-0111769-9.

6. Rukunuzzaman M, Karim MB, Rahman MM, et al. Wilson's disease in children with blindness: an atypical presentation. Mymensingh Med J 2013;22(1):176-179.

7. Karim MB, Rahman MM, Islam MS. Wilson's disease with hepatic presentation in childhood. Mymensingh Med J 2007;16(1):29-32. DOI: 10.3329/mmj.v16i1.244.

8. Rahman S, Siddiqui NI, Paul GK, et al. A case report on Wilson's disease. Mymensingh Med J 2003;12(2):142-145.

9. Rukunuzzaman M. Wilson's disease in Bangladeshi children: analysis of 100 cases. Pediatr Gastroenterol Hepatol Nutr 2015;18(2):121-127. DOI: 10.5223/pghn.2015.18.2.121.

10. Alam ST, Rahman MM, Islam KA, et al. Neurologic manifestations, diagnosis and management of Wilson's disease in children - an update. Mymensingh Med J 2014;23(1):195-203.

11. Ullah AKMA, Maksud MA, Khan SR, et al. Morning (first) urine copper concentration: a new approach for the diagnosis of Wilson's disease. Biol Trace Elem Res 2019;190(2):283-288. DOI: 10.1007/s12011-0181547-z.

12. Ala A, Walker AP, Ashkan K, et al. Wilson's disease. Lancet 2007;369(9559):397-408. DOI: 10.1016/S0140-6736(07)60196-2.

13. Taly $A B$, Meenakshi-Sundaram $S$, Sinha $S$, et al. Wilson disease: description of 282 patients evaluated over 3 decades. Medicine (Baltimore) 2007;86(2):112-121. DOI: 10.1097/MD.0b013e318045a00e.

14. Mak LY, Cruz-Ramón V, Chinchilla-López P, et al. Global epidemiology, prevention, and management of hepatocellular carcinoma. Am Soc Clin Oncol Educ Book 2018;38:262-279. DOI: 10.1200/EDBK_200939.

15. Devarbhavi $H$, Singh R, Adarsh CK, et al. Factors that predict mortality in children with Wilson disease associated acute liver failure and comparison of Wilson disease specific prognostic indices. J Gastroenterol Hepatol 2014;29(2):380-386. DOI: 10.1111/jgh.12356.

16. Liu J, Luan J, Zhou X, et al. Epidemiology, diagnosis, and treatment of Wilson's disease. Intractable Rare Dis Res 2017;6(4):249-255. DOI: 10.5582/irdr.2017.01057.

17. Taly $A B$, Prashanth LK, Sinha S. Wilson's disease: an Indian perspective. Neurol India 2009;57(5):528-540. DOI: 10.4103/0028-3886.57789. 